Why is the Schwarzschild Solution Independent of the Spatial Stresses?

S. Deser and B. E. Laurent

Citation: 36, (1968); doi: 10.1119/1.1975149

View online: http://dx.doi.org/10.1119/1.1975149

View Table of Contents: http://aapt.scitation.org/toc/ajp/36/9

Published by the American Association of Physics Teachers 


\title{
Why is the Schwarzschild Solution Independent of the Spatial Stresses?*
}

\author{
S. Deser and B. E. Laurent \\ Physics Department, Brandeis University, Waltham, Massachusetts 0\%154
}

(Received 8 May 1968)

\begin{abstract}
The following paradox is raised and resolved: Both the energy density $T_{00}$ and the spatial stresses $T_{k l}$ are (independent) sources of the tensor gravitational field in general relativity. Yet the general Schwarzschild exterior solution depends only on one parameter $m$, related to $T_{00}$, so that no properties of $T_{i j}$ "propagate" to affect an outside observer.
\end{abstract}

\section{INTRODUCTION}

We raise, and then resolve, an apparent paradox in the simplest solution of general relativitythe exterior spherically symmetric Schwarzschild metric. As is well known, Birkhoff's theorem states that the metric may be put in the standard Schwarzschild form outside any spherically symmetric source. The single total mass parameter $m$ thus represents all properties of the interior matter distribution relevant to an outside observer. Were this not the case, there could be an essential difference from Newtonian theory outside such sources. Thus this tensor theory reduces, in the spherically symmetric case, to an effective scalar Poisson-like equation. On the other hand, a source, even if static and spherically symmetric, may (as we see) possess spatial stresses ${ }^{1} T_{k l}(r)$, in addition to, and independent of, its energy density $T_{00}(r)$. Why then do none of the properties of $T_{k l}$ affect the exterior metric through the $G_{l k}={ }_{k} T_{l k}$ equations just as $T_{00}$ determines $G_{00}$ ?

For simplicity, our discussion will be mainly within the framework of the linearized approximation, where the essentials are the same as in the full theory.

\section{ELECTRODYNAMICS}

It may be instructive, for later comparison, to note first why no corresponding problem arises in a vector theory such as electrodynamics. Here, current conservation reads $\partial_{\mu} j^{\mu}=0=\partial_{0} j^{0}+\nabla^{2} j=0$, using spherical symmetry, which implies $j^{i}(r, t) \equiv$

\footnotetext{
* Supported in part by U. S. Air Force OAR, Grant OSR 368-67.

$\dagger$ On leave from University of Stockholm, Stockholm, Sweden.

1 These are, of course, not to be confused with an energy quadrupole moment, which is necessarily absent by spherical symmetry.
}

$\partial_{i} j(r, t)$; the scalar function $j$ is then determined by the charge density; thus $j$ vanishes if there is no time dependence. Equivalently, we know that the necessarily purely longitudinal vector potential, $A^{i}(r, t) \equiv \partial_{i} a(r, t)$, is therefore a gauge; the scalar potential $A^{0}$, or the gauge-invariant contribution $F_{0} \equiv\left(\partial_{0} a+A^{0}\right)$ is the only variable in the problem. The latter is determined entirely by $j^{0}$. Alternately, the spatial component of the Maxwell equations reads

$$
\partial_{i}\left[\partial_{0} F_{0}-j(r, t)\right]=0
$$

and, neglecting the trivial integration factor, we have $\partial_{0} F_{0}=j(r, t)$. This is a spatially local relation, as against the Poisson equation $\nabla^{2} F_{0}=-j^{0}$, so that, outside the charges, $F_{0}$ is unaffected by the current density. ${ }^{2}$

\section{GRAVITATION}

We turn now to the spin-2 gravitational theory. It is well known that the coupling of a dynamical source to the linearized field is inconsistent (the conservation requirement $\partial_{\mu} T^{\mu \nu}=0$ on the source's stress tensor is violated by the interaction with the field); however (conserved) prescribed sources are permitted, and are sufficient for our purpose.

Consider first the exterior region, where the (linearized) empty-space equations hold:

$$
\begin{aligned}
& G_{\mu \nu}{ }^{(L)} \equiv h_{\mu \nu}-h_{\mu}{ }^{\alpha}{ }_{, \alpha \nu}-h_{\nu}{ }^{\alpha}{ }_{, \alpha \mu}+h_{\alpha}{ }^{\alpha}{ }_{, \mu \nu} \\
& -\eta_{\mu \nu}\left(h_{\alpha}{ }^{\alpha}-h^{\alpha \beta}{ }_{\alpha \beta}\right)=0 .
\end{aligned}
$$

2 There are, of course, similarities to spin-2 theory, primarily the existence of a Birkhoff theorem in both cases. Its content here is that there exist gauges (e.g., $a=0$ ), in which the exterior solution is static. This is a property peculiar to the transverse nature of the fields and would not hold for, say, a scalar field, which can be radiated by a spherically symmetric source [that is, $\square \phi(r, t)=\rho(r, t)$ implies an exterior time-dependent $\phi$ field]. 
Here $h_{\mu \nu}$ is the spin 2 field and all index shifts are earred out with the Lorent/ metric $\eta_{\mu \nu}(+++-)$. Note the identity $\left(f^{(s) \mu \nu}, \nu \equiv 0\right.$, and the invarianer of the equations under $h_{\mu \nu} \rightarrow h_{\mu \nu}+\xi_{\mu, \nu}+\xi_{p, \mu}$.

Since we are not interested in rederiving the time independence of the exterior solution, let us simplify by assuming the appropriate gauge choice to have been made so that the amplitude $h_{\mu \nu}$ is time independent. The equations then read

$$
\begin{aligned}
& G_{00}=h_{k k, l l}-h_{k l, k l l}=0, \\
& G_{0 i}=\nabla^{2} h_{0 i}-h_{0 k, k i}=0, \\
& \begin{aligned}
G_{i j}=\nabla^{2} h_{i j}-h_{i k, k j}-h_{j k_{k} k i}+\left(\partial_{i j}{ }^{2}-\delta_{i j} \nabla^{2}\right)\left(h_{k l i}-h_{00}\right) \\
\quad+\delta_{i j} h_{l m, l m}=0 .
\end{aligned}
\end{aligned}
$$

Spherical symmetry allows us to write the metric as follows:

$$
\begin{aligned}
& h_{00} \equiv N(r), \quad h_{0 i} \equiv \partial_{i} f(r), \\
& h_{i j} \equiv \delta_{i j} h(r)+\partial_{i j}{ }^{2} \beta(r) .
\end{aligned}
$$

The form (3) of $h_{i j}$ is equivalent to, but more convenient than, the usual $\delta_{i j} A(r)+x^{i} x^{j} B(r)$; the argument would, of course, go through with the latter as well. The functions $f(r)$ and $\beta(r)$ are, by their definition, pure gauges, and so never appear in the gauge-invariant field equations. The two remaining functions, $N\left(r^{\prime}\right)$ and $h(r)$ are determined by:

$$
\begin{aligned}
& G_{00}=2\left(\nabla^{2} h\right)=0, \\
& G_{i j}=\left[\nabla^{2} \delta_{i j}-\partial^{2}{ }_{i j}\right](N-h)=0 .
\end{aligned}
$$

The spatial metric is then of the form $h=m / r$, by (4a). Taking the trace of (4b), we obtain $\nabla^{2}(N-h)=0$, which at first sight permits $N$ to involve an independent parameter, $N=m^{\prime} / r$. Inserting this into (4b), however, we have $\left(m^{\prime}-m\right) \partial_{i j}{ }^{2} 1 / r=0$ which requires that $m^{\prime}=m$, and so $N=h$. From this exterior point of view, the appearance of the single parameter $m$ is traceable to the fact that only $G_{00}=0$ is a Laplace equation. The $G_{i j}=0$ equations are of the stronger form $\partial_{i j}{ }^{2} \phi=0$ which has no homogeneous solutions vanishing at infinity. The content of this result is that there cannot be a free spin 2 (or spin 1) transverse structure with spherical symmetry. The spatial equations (2c) which govern the hehavior of the spin-2 amplitudes are, in their spherically symmetric form (6), effectively statements of this fact. This completes the derivation. It is instructive, however, to follow the derivation also when the sources are taken into account explicitly. With the static assumption ${ }^{3}$ the conservation laws read $T^{0 i}{ }_{i}=0=T^{i j}{ }_{i}$. The momentum density must, of course, vanish in a static

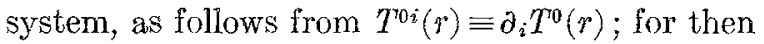
conservation implies that the scalar function $T^{0}$ obeys $\nabla^{2} T^{0}(r)=0$ and so vanishes. On the other hand, $T^{00}(r)$ and $T^{i j}(r)$ are not fully determined by conservation. The former is left arbitrary, as is one function in $T_{i j}$. For spherical symmetry implies $T_{i j} \equiv A(r) \delta_{i j}-\partial_{i j}{ }^{2} T(r)$, and $T^{i j}{ }_{j}=0$ then yields $A=\nabla^{2} T(r)$. Thus, we have

$$
T_{i j}(r)=\left(\nabla^{2} \delta_{i j}-\partial_{i j}{ }^{2}\right) T(r),
$$

with $T(r)$ arbitrary. Equation (4b) now takes the form

$$
\left(\nabla^{2} \delta_{i j}-\partial_{i j}{ }^{2}\right)\left[N-h-{ }_{k} T(r)\right]=0
$$

which shows, from the interior source viewpoint, why there is no effect from $T(r)$ outside the body, ${ }^{4}$ since (6) implies $N-h=\kappa T(r)$. For, the space-local structure $\left(\nabla^{2} \delta_{i j}-\partial_{i j}{ }^{2}\right)$ or more relevantly, $\partial_{i j}{ }^{2}$, acts on both field and source, in contrast to the $\nabla^{2} \phi=\rho$ Poisson case. Thus, the longitudinal part of the field has effectively one component (in the static frame--four in general) and so can only propagate $m$ (or $P_{\mu}$ ) but not $T_{i j}$.

While the full nonlinear theory is formally more complicated, the results and reasoning are similar. We merely sketch the procedure: The exterior equations $G_{00}=0, G_{i j}=0$ read, in the static case $\left(\dot{g}_{\mu \nu}=0=g_{0 i}\right)$,

$$
\begin{aligned}
{ }^{3} R & =0 \\
N\left({ }^{3} R_{i j}-\frac{1}{2} g_{i j}{ }^{3} R\right) & =\left(\nabla_{i} \nabla_{j}-g_{i j} \nabla_{l} \nabla^{l}\right) N,
\end{aligned}
$$

where ${ }^{3} R_{i j}$ is the Ricci tensor of the spatial metric $g_{i j}$ and ${ }^{3} R \equiv{ }^{3} R_{l}{ }^{l} ; N^{2} \equiv-g_{00}$ and $\nabla_{i}$ is the covariant

\footnotetext{
${ }^{3} \mathrm{Had}$ we kept a time-dependent source, there would have been no essential difference in the diseussion.

4 The important point here is that the function $T(r)$ is locally related to the source's stress tensor and vanishes when $T_{i f}$ does. This would not be the case if we had, for example, $T_{i j}(r)=\delta_{i j} \nabla 2 T(r)$, for then $T(r)$ could have the form (const)/r outside the matter.
} 
derivative with respect to $g_{i j}$. Condition (6a) is essentially a Laplace equation determining $g_{i j}$. For, as in the linear case, spherical symmetry implies that $g_{i j}$ depends on two functions, one of which is a gauge. [For example, in isotropic coordinates, $g_{i j}=\chi^{4}(r) \delta_{i j}$ which gives

$$
\left(\partial_{x}{ }^{2}+\partial_{y}{ }^{2}+\partial_{z}{ }^{2}\right) \chi=0
$$

and so $\chi=1+m / 2 r$.] Taking the trace of Eq. (6b), we conclude that $\nabla_{l} \nabla^{l} N=0$; hence, $N$ satisfies $\nabla_{i} \nabla_{j} N=N^{3} R_{i j}$. This equation has two free derivatives and so determines $N$ completely in terms of $g_{i j}$. [In the isotropic case one obtains
$N=2 \chi^{-1}-1$, i.e., the familiar form

$$
\left.-g_{00}=\left(\frac{1-(m / 2 r)}{1+(m / 2 r)}\right)^{2} \cdot\right]
$$

Explicit inclusion of sources in the nonlinear case is again unnecessary, but when they are taken into account, $T_{i j}$ is seen (as in the linear theory) to affect only the interior metric.

Finally, we emphasize that the $T_{i j}$ independence of the exterior solution is specific to the pure spin-2 theory; admixture of spin 0, for example, will generally lead to explicit exterior dependence on the interior spatial stress.

\title{
A Simplified Derivation of the Irreducible Representations of $S U(3)^{*}$
}

\author{
T. J. NELSON \\ Institute for Atomic Research and Department of Physics, Iowa State University, Ames, Iowa 50010 \\ (Received 9 May 1968)
}

\begin{abstract}
A simple solution to the eigenvalue problem posed by a set of operators which have the commutation rules of $S U(3)$ is developed. The analogy with the more familiar problem for $S U(2)$, i.e., angular momentum, is used as a guide. The set of labels used to describe the states in an ixreducible representation admits of a folded-ladder interpretation that is particularly transparent. Finally the dimensionality formula and the effect of complex conjugation are derived.
\end{abstract}

\section{INTRODUCTION}

The purpose of this article is to give a simple solution to the eigenvalue problem posed by a set of operators which have the commutation rules of what is known in group theory as the Lie algebra of $S U(3)$. The solution of this problem is already well known. The technique to be used is essentially that of Biedenharn ${ }^{1}$ although the notation to be employed most closely resembles that of Pursey. ${ }^{2} \mathrm{~A}$ third derivation that relies on the development of a set of partial differential equations as an intermediate step has been given by Nelson. ${ }^{3}$

* Work was performed in the Ames Laboratory of the U. S. Atomic Energy Commission. Contribution No. 2316.

${ }^{1}$ L. C. Biedenharn, Lectures in Theoretical Physics W. E. Britin, B. W. Downs, and J. Downs, Eds. (Interscience Publishing, Inc., New York, 1963), Vol. 5, p. 258.

${ }^{2}$ D. L. Pursey, Proc. Roy. Soc. (London) A275, 284 (1963).

' T. J. Nelson, J. Math. Phys. 8, 857 (1967)。
The group $S U(3)$ is of interest in physics because it has been possible to classify isotopic spin-hypercharge submultiplets of particles with some of its irreducible representations. Because only a few of the simplest representations have been needed for this purpose, the general $S U(3)$ state has not been understood as well as it might be. In the next section, the standard problem for $S U(2)$ is briefly reviewed. This provides some of the results and notation needed in the treatment of $S U(3)$. However, a more important reason for discussing irreducible representations of $S U(2)$ is that this provides an example of the method to be used in the solution of the more complicated problem, and also of the significance for group theory of the steps in the derivation. The algebra for $S U(3)$ is then discussed and solved. Finally, a discussion of the irreducible representations of $S U(3)$ based on the results attained is made, and it is shown that these representations are actually as easy to understand as are those of $S U(2)$. 